\title{
Fluctuations of anticardiolipin antibody levels in patients with systemic lupus erythematosus: a prospective study
}

\author{
HENK JAN OUT, ${ }^{1}$ PHILIP G DE GROOT ${ }^{2}$ PAULA HASSELAAR, ${ }^{1}{ }^{2}$ \\ MARJA VAN VLIET, ${ }^{2}$ AND RONALD H W M DERKSEN \\ From the 'Division of Immunopathology (Department of Internal Medicine) and the ${ }^{2}$ Department of \\ Hematology, University Hospital Utrecht, The Netherlands
}

SUMMARY In 53 patients with systemic lupus erythematosus sequential blood samples obtained during a four year period (range 6-47 months) were screened for anticardiolipin antibodies (ACAs). Disease activity and treatment with prednisone were also assessed and related to ACA concentrations. During follow up only 21 patients for ACA IgG (40\%) and 25 for ACA IgM $(47 \%)$ remained in the ACA category (negative, low positive, high positive) found at the first sample taken at entrance. Marked increases from negative to high positive concentrations were sometimes seen and were not accompanied by typical events such as thrombosis or thrombocytopenia (the ACA syndrome). Shifts in ACA concentrations could not always be explained by changes in prednisone dose. Also, in patients with low dose prednisone treatment or none at all $(n=22) 10$ patients $(45 \%)$ changed ACA IgG category and 12 patients $(55 \%)$ fluctuated in ACA IgM categories during follow up. As a consequence of the variability in ACA titres relations of ACAs with the ACA syndrome depended on the blood sample studied. In the second sample, randomly taken half way through follow up, no significant relations with the ACA syndrome could be found. Anticardiolipin antibody IgG was significantly associated with disease activity in $11 / 47$ patients $(23 \%)$ and in the group as a whole. During remission ACA IgG was significantly associated with the ACA syndrome, whereas during moderate/severe disease activity in the same patients that correlation was not significant. Anticardiolipin antibody IgM was much less influenced by disease activity, and in only $4 / 47$ patients ( $9 \%$ ) could a significant relation with disease activity be shown. Associations of ACA IgM with the ACA syndrome were significant during both lupus flares and remission.

Systemic lupus erythematosus (SLE) is a multisystem autoimmune disease characterised by a variety of circulating autoantibodies. Of these, antibodies against negatively charged phospholipids, such as anticardiolipin antibodies (ACAs), have received a lot of attention over the past decade. Many studies showed correlations between the presence of ACAs and particular clinical features, such as fetal loss, thrombosis, and thrombocytopenia. ${ }^{1-5}$ The term 'anticardiolipin syndrome' has been introduced for this entity. ${ }^{6}$

Longitudinal data on ACAs are scarce. Suggestions have been made that ACA titres may vary with

Accepted for publication 6 April 1989.

Correspondence to Dr H J Out. Division of Immunopathology, Department of Internal Medicine. University Hospital Utrecht. PO Box 85500, 3508 GA. Utrecht. The Netherlands. disease activity ${ }^{7}$ and that they may disappear spontaneously. ${ }^{9}$ It has been shown that immunosuppressive drugs influence ACA concentrations. ${ }^{10}$

Relations between ACAs and the ACA syndrome are usually established in cross sectional studies. Also, classification of patients as ACA positive or negative is based on single blood samples. To investigate whether such classification is valid we prospectively studied 53 patients with SLE over a four year period (range 6-47 months) and focused on the relation between ACA titres, lupus disease activity, and prednisone treatment.

\section{Patients and methods}

PATIENTS AND SAMPLES

Clinical data and blood samples of 53 patients with 
SLE, according to American Rheumatism Association criteria, ${ }^{11}$ were collected from September 1984 until August 1988 at each visit to our outpatient clinic and during hospital admission. There were 49 women (median age 36 years, range 18-67) and four men (median age 33 years, range $30-37$ ). The median duration of follow up was 26 months (range

Table 1 Systemic lupus erythematosus activity scoring system*

Activity
$\begin{aligned} & \text { Renal } \\ & \text { Proteinuria }>0 \cdot 5 \mathrm{~g} / 24 \text { hours (onset within }+ \text { months } \\ & \text { or duplication) } \\ & \text { Haematuria (onset or duplication, }>5 \mathrm{RBC} \div / \mathrm{HPF} \div \text { ) } \\ & \text { Casts (red blood cell or granular): presence } \\ & \text { onset }\end{aligned}$
Creatinine clearance: drop $>25 \%$, within 4 months
Cerebral
Cerebrovascular accident
Insults
Psychosis
Choreathetosis
Brain nerve paralysis

Skin/hair/mucous membranes

Alopecia

Active discoid lesions

Butterfly rash

Other active rashes

Active oral ulceration

Joints

Arthralgia or myalgia, or both

Arthritis ( $\geqslant 2$ joints) or tendinitis, or both

Score

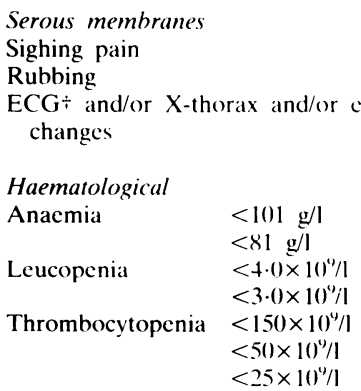

Vascular

Minor vasculitis (purpura, periungual infarcts)

Major vasculitis (ulcers, mononeuritis)

Miscellaneous

Uveitis or chorioretinitis. or both

Myositis: $\mathrm{CPK} \div>150 \mathrm{U} / \mathrm{l}$

$$
>5(x) \mathrm{U} / \mathrm{l}
$$

${ }^{*}$ Only active lesions or new onset of symptoms are scored. $\div \mathrm{RBC}=$ red blood cells: $\mathrm{HPF}=$ high powered field: $\mathrm{ECG}=$ electrocardiogram: $(\mathrm{PK}=$ creatine phosphokinase.
6-47 months), during which at least seven bloof samples (range 7-22, median 12 samples) were. taken. Blood samples for the preparation of seruncs were collected by venepuncture into glass tubeso The blood was allowed to clot at room temperature for two hours and centrifuged at $175 \mathrm{~g}$ for 10 minutes to obtain serum.

DISEASE ACTIVITY

Disease activity and prednisone treatment were recorded at each sample point. Disease activity was assessed with a scoring system consisting of typicab clinical and laboratory features of SLE (Table 1 1 and classified as inactive (scores $0-1$ ), mild (score $2-3$ ), moderate (score $4-5$ ), or severe (score $\geqslant 6$ )...

Table 2 shows lupus symptoms at entrance and during follow up, classified according to organ sys $\overrightarrow{-}$ tem. The most common manifestations of active dis N ease were arthritis, cytopenia, symptoms of the skiro and mucous membranes. Sixteen patients had rena? disease.

ANTICARDIOLIPIN ANTIBODY ASSAY Anticardiolipin antibodies (IgG and IgM) were measured with an enzyme linked immunosorbent assay (ELISA) according to the procedure descritget by Loizou et al ${ }^{12}$ with minor modifications. ${ }^{13} \mathrm{~A}$ pesio tive result indicated a value in units more than 290 over the value obtained with pooled control serump derived from 71 healthy volunteers. All results weres expressed as the ratio of patient ACA concentra응 tions to that of normal values plus $2 \mathrm{SD}$ in order to make comparisons of ACA assays performed on dif ferent days and to make calculations of mean value $\overrightarrow{\vec{D}}$ for whole group analysis. A ratio $<1$ represented negative ACA concentrations, values between 1 an 2 were regarded as low positive, and ratios of more than 2 indicated high positive ACA titres.

RELATION BETWEEN ACAS AND THE

ANTICARDIOLIPIN SYNDROME

Correlations of ACA IgG and ACA IgM for fetą

Table 2 Lupus symptoms of 53 patients with systemi lupus ervthematosus at entrance and during follow up. classified according to organ system

\begin{tabular}{lc}
\hline Symptoms & Patients $(n)$ \\
\hline Renal & 16 \\
Cerebral & 6 \\
Skin/hair/mucous membranes & 35 \\
Joints & 34 \\
Haematological & 33 \\
Serous membranes & 7 \\
Vascular & 17 \\
Miscellaneous & 2 \\
\hline
\end{tabular}

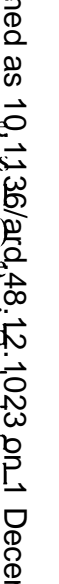


Anticardiolipin antibody levels in patients with SLE 1025

Table 3 Number of patients (\%) in each anticardiolipin antibody (ACA) and disease activity category at the first sample

\begin{tabular}{|c|c|c|c|c|c|c|c|}
\hline \multirow{2}{*}{$\begin{array}{l}\text { Disease } \\
\text { Activity }\end{array}$} & \multicolumn{3}{|c|}{$A C A \quad I g G$} & \multirow[t]{2}{*}{ Total } & \multicolumn{3}{|c|}{$A C A \quad \operatorname{Ig} M$} \\
\hline & Neg & Low pos & High pos & & $\mathrm{Neg}$ & Low pos & High pos \\
\hline $0-1$ (none) & 13 & 6 & 3 & $22(42)$ & 17 & 3 & 2 \\
\hline 2-3 (mild) & 11 & 6 & 3 & $20(38)$ & 12 & 5 & 3 \\
\hline 4-5 (moderate) & 4 & 1 & 2 & 7 (13) & 6 & 1 & 0 \\
\hline$\geqslant 6$ (severe) & 1 & 3 & 0 & $4(7)$ & 2 & 1 & 1 \\
\hline Total & $29(55)$ & $16(30)$ & $8(15)$ & $53(100)$ & $37(70)$ & $10(19)$ & $6(11)$ \\
\hline
\end{tabular}

loss, thrombosis, and thrombocytopenia were assessed at three random points: at the start, half way through follow up, and the last taken sample. Also, comparisons of correlations with the ACA syndrome were made using the first samples in both no disease/mild disease and moderate/severe activity, in 32 patients who had had at least one lupus flare.

\section{ST A T IS T I CS}

Correlations within each patient between disease activity, ACA IgG, and ACA IgM were made with Pearson's correlation coefficient. Correlations of ACAs with features of the ACA syndrome were calculated using the $\chi^{2}$ test. To investigate the interrelations between ACAs and disease activity for the studied population as a whole six measure points of each patient were selected from the available data using a table with random numbers. For this whole group analysis $\chi^{2}$ tests were done. Values of $p<0.05$ were considered significant.

\section{Results}

FIRST SAMPLE

Twenty four patients $(45 \%)$ were positive for ACA IgG and 16 patients $(30 \%)$ had positive ACA IgM concentrations (Table 3). Most patients (42/53 $(80 \%))$ had mild disease activity or none at all. Four patients received more than $40 \mathrm{mg}$ prednisone (Table 4). Of these, three patients were positive for ACA IgG and two for ACA IgM.
FOLLOW UP

Table 5 shows the ACA concentrations of each patient during follow up compared with the ACA category found at the first sample. When all patients together are considered $13 / 29$ patients $(45 \%)$ with negative ACA IgG samples at entrance and 14/37 (38\%) with initially negative ACA IgM concentrations developed (low) positive samples during follow up. For both ACA IgG and ACA IgM two patients developed high positive samples. Similarly, 14/24 patients $(58 \%)$ starting with positive ACA IgG concentrations and $7 / 16(44 \%)$ with positive ACA IgM concentrations at entrance showed negative samples during the course of the disease.

Fluctuations were also observed in patients with no or mild disease activity $(n=20)$ : three of 10 negative patients $(30 \%)$ became positive (of which one high positive) for ACA IgG and 6/15 negative patients $(40 \%)$ had positive (including one high positive) ACA IgM concentrations during follow up. Five of 10 initially ACA IgG positive patients $(50 \%)$ showed negative samples during follow up. In all four patients starting with high positive ACA samples and with no disease/mild disease activity during follow up ACA titres remained positive.

To exclude variations in prednisone dose as the cause for ACA shifts all patients with no $(n=10)$ or low dose $(\leqslant 10 \mathrm{mg})$ prednisone $(n=12)$ treatment were evaluated separately. Of nine ACA IgG negative patients at entrance, three $(33 \%)$ became positive, whereas six of $12(50 \%)$ ACA IgM negative

Table 4 Prednisone treatment at the first sample

\begin{tabular}{|c|c|c|c|c|c|c|}
\hline \multirow{2}{*}{$\begin{array}{l}\text { Prednisone } \\
\text { (mg) }\end{array}$} & \multicolumn{3}{|c|}{$A C A^{*} \lg G$} & \multicolumn{3}{|c|}{$A C A \quad I g M$} \\
\hline & Neg & Low pos & High pos & $\mathrm{Neg}$ & Low pos & High pos \\
\hline None & 8 & 7 & 3 & 13 & 2 & 3 \\
\hline$\leqslant 10$ & 8 & 4 & 3 & 9 & 5 & 1 \\
\hline $10-40$ & 12 & 2 & 2 & 13 & 2 & 1 \\
\hline$>40$ & 1 & 3 & 0 & 2 & 1 & 1 \\
\hline Total & 29 & 16 & 8 & 37 & 10 & 6 \\
\hline
\end{tabular}

${ }^{*} \mathrm{ACA}=$ anticardiolipin antibody. 
Table 5 Number of patients in each anticardiolipin antibody (ACA) category during follow up

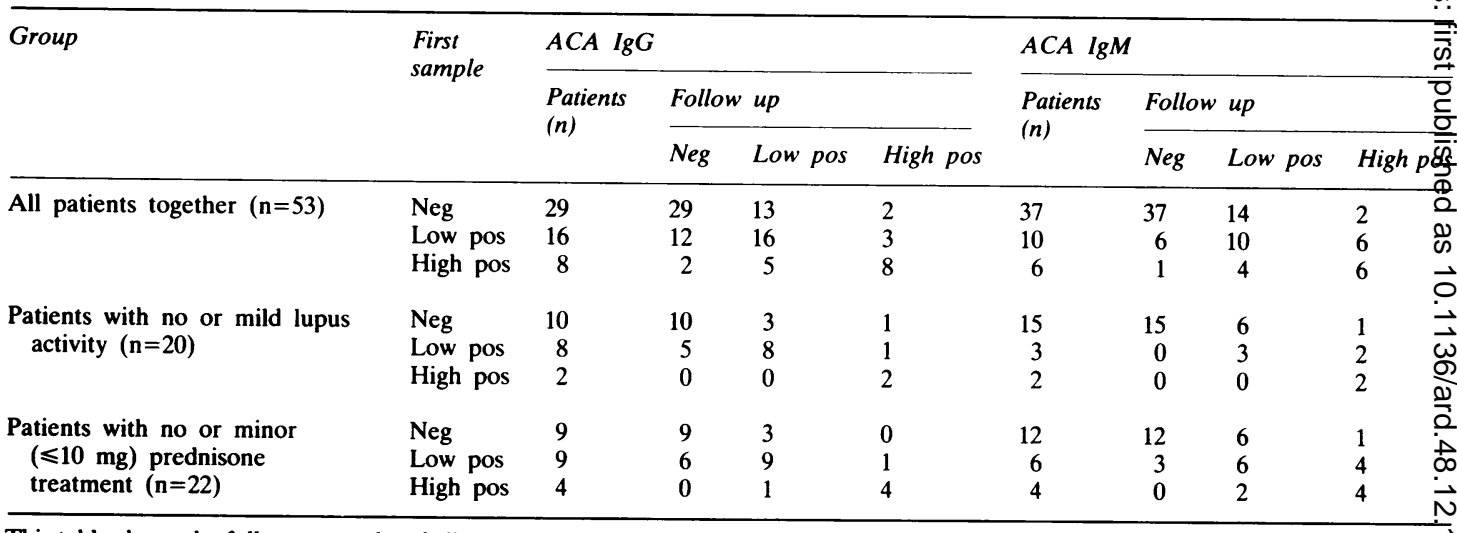

This table shows the follow up results of all patients classified as negative, low positive, or high positive at the first sample. The results are given as the number of patients with samples in each ACA category, including the first sample. As during follow up one patient could hat samples classified in more than one ACA category the total number of patients obtained by adding all categories exceeds the number at the first sample.

patients developed positive titres, including one patient with high positive levels. Six of nine $(67 \%)$ initially low positive patients became negative for ACA IgG, and $3 / 6(50 \%)$ for ACA IgM.

\section{CORRELATION OF ACAS AND DISEASE \\ ACTIVITY}

Anticardiolipin antibody IgG and disease activity were significantly correlated in 11 of 47 patients $(23 \%)$; six patients with persistently negative ACA IgG concentrations and absent lupus activity were excluded because in those circumstances associations would be expected. Significant correlations between ACA IgM and disease activity were found in only four of 47 patients $(9 \%)$ with fluctuating ACA IgM concentrations or lupus activity, of both.

Anticardiolipin antibody IgG and ACA IgM were correlated in 17 of 41 patients $(41 \%)$, excludingalfip patients with persistently negative ACA IgG ân ACA IgM concentrations $(n=12)$. Figure 1 illus trates graphically the relation between ACA IgGACA IgM, prednisone treatment, and disease activő ity in two female patients with SLE. In one patieng ACAs and disease activity were correlated signifi cantly, whereas the other patient showed a drop o\$ high positive ACA IgG concentrations to normat during no disease/mild disease activity.
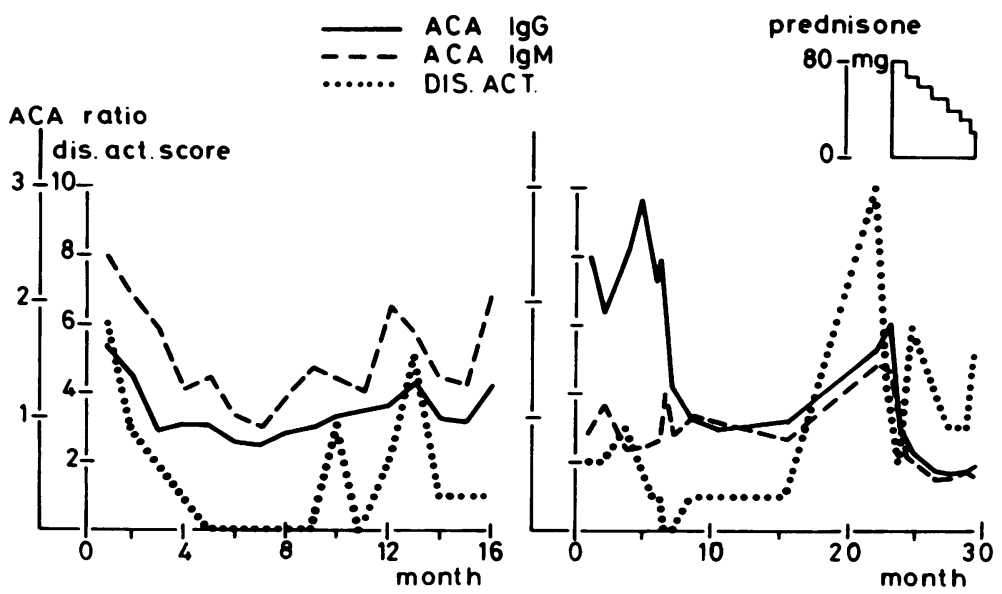

Fig. 1 Follow up results for two female patients with systemic lupus erythematosus. Left: Anticardiolipin antibody ( $A C A)$ $\operatorname{IgG}, A C A$ IgM, and disease activity are correlated significantly 을 $(p<0.02)$. No prednisone was given. Anticardiolipin antibody ratios $>1$ are positive, $A C A$ ratios $N$ $>2$ are regarded as high positive. Right: No significant correlations. Initial spontaneous drop in $A C A$ IgG concentrations from high positive to negative values. Normak ACA IgG concentrations are seen? when high dose prednisone is given. 
Table 6 Correlation of anticardiolipin antibody (ACA) $\operatorname{IgG}$ and ACA IgM (in p values, $\chi^{2}$ ) with the ACA syndrome at different sample points in 53 patients with systemic lupus erythematosus

\begin{tabular}{|c|c|c|c|c|c|c|}
\hline \multirow{2}{*}{$\begin{array}{l}\text { Sample* } \\
\text { point }\end{array}$} & \multicolumn{3}{|c|}{$A C A \operatorname{Ig} G$} & \multicolumn{3}{|c|}{$A C A \operatorname{Ig} \dot{M}$} \\
\hline & $\begin{array}{l}\text { Fetal }+ \\
\text { loss }\end{array}$ & Thrombosis & Thrombocytopenia & $\begin{array}{l}\text { Fetal } \\
\text { loss }\end{array}$ & Thrombosis & Thrombocytopenia \\
\hline $\begin{array}{l}\text { First sample } \\
\text { Second sample } \\
\text { Third sample }\end{array}$ & $\begin{array}{l}0 \cdot 035 \\
\text { NS } \\
\text { NS }\end{array}$ & $\begin{array}{l}0 \cdot 008 \\
\text { NS } \\
0.026\end{array}$ & $\begin{array}{l}0.008 \\
\text { NS } \\
\text { NS }\end{array}$ & $\begin{array}{l}0 \cdot 010 \\
\text { NS } \\
\text { NS }\end{array}$ & $\begin{array}{l}0 \cdot 002 \\
\text { NS } \\
0 \cdot 019\end{array}$ & $\begin{array}{l}0.005 \\
\text { NS } \\
\text { NS }\end{array}$ \\
\hline $\begin{array}{l}\text { First sample taken } \\
\text { No/mild dis.act. } \\
\text { Mod./sev. dis.act. }\end{array}$ & $\begin{array}{l}\text { ing: } \\
\text { TSN } \ddagger \\
\text { TSN }\end{array}$ & $\begin{array}{l}0 \cdot 003 \\
\text { NS }\end{array}$ & $\begin{array}{l}\text { NS } \\
\text { NS }\end{array}$ & $\begin{array}{l}\text { TSN } \\
\text { TSN }\end{array}$ & $\begin{array}{l}0.013 \\
0.033\end{array}$ & $\begin{array}{l}\text { NS } \\
\text { NS }\end{array}$ \\
\hline
\end{tabular}

*First sample taken at the start of the study; second sample taken half way through follow up; third sample taken at the end of the study. $\dagger$ Twenty seven patients had a history of at least one pregnancy.

$\ddagger T S N=$ numbers too small for statistical analysis $(n=13)$.

RELATION OF ACAS WITH THE

ANTICARDIOLIPIN SYNDROME

Table 6 gives the relations between major manifestations of the ACA syndrome and ACAs at different random sample points.

With the first sample significant relations between ACAs and all features of the ACA syndrome were found. The second sample, however, taken half way through follow up did not show any significant relations at all. With the third sample an association only with thrombosis was found and not with fetal loss or thrombocytopenia.

When ACA concentrations were considered in the first sample obtained during no disease/mild disease activity and compared with ACA titres in the same patients during moderate/severe lupus activity $(n=32)$, ACA IgG was not significantly associated with the ACA syndrome during lupus flares, whereas at remission ACA IgG showed a relation with thrombosis $(p=0.003)$. For ACA IgM differences in disease activity did not influence significant relations.

Table 7 Whole group analysis. Mean values of anticardiolipin antibody ( $A C A) \operatorname{IgG}$ and $A C A \operatorname{IgM}$ at each category of disease activity

\begin{tabular}{lllll}
\hline \multicolumn{5}{c}{ Disease activity } \\
& None & Mild & Moderate & Severe \\
\hline ACA IgG* & 1.4943 & 1.2585 & 1.3196 & 1.8886 \\
ACA IgM ${ }^{+}$ & 1.5998 & 1.1566 & 0.9814 & 0.9494 \\
\hline
\end{tabular}

* $p=0.012\left(\chi^{2}\right)$ correlation between disease activity and ACA IgG. $+\mathrm{p}=0.197\left(\chi^{2}\right)$ correlation between disease activity and ACA IgM. Anticardiolipin antibody IgG and ACA IgM were correlated significantly $\left(p<0 \cdot 001, \chi^{2}\right)$.
WHOLE GROUP ANALYSIS

Table 7 shows the results of whole group analysis, based on six random samples from each patient. $\chi^{2}$ Analysis showed significant relations between ACA IgG and disease activity $(p=0.012)$, and between ACA IgG and ACA IgM $(p<0.001)$, but not between disease activity and ACA IgM $(p=0 \cdot 197)$.

\section{Discussion}

This prospective study of 53 patients with SLE shows the occurrence of shifts in ACA concentrations. During follow up only 21 patients $(40 \%)$ for ACA IgG and 25 (47\%) for ACA IgM remained in the ACA category (negative, low positive, high positive) initially found. Marked increases from negative to high positive concentrations were sometimes observed (Fig. 1) and were not accompanied by symptoms like thrombosis or thrombocytopenia. High dose prednisone may explain the variability in ACA concentrations (Fig. 1), but in patients with low dose prednisone treatment or none at all 10 patients (45\%) changed ACA IgG category and 12 patients $(55 \%)$ fluctuated in ACA IgM categories (Table 5). Because of the variability of ACA concentrations the time of evaluation affects the significance of the relation between ACAs and features of the ACA syndrome (Table 6).

These findings may explain the conflicting results found by various investigators for the prevalence of ACAs in SLE, ranging from $22 \%^{14}$ to $38 \%{ }^{15}$ to $54.2 \%,{ }^{16}$ and the subsequent interrelations of ACAs with the ACA syndrome. Weidmann et al did not find any association between ACAs and thrombosis or spontaneous abortions. ${ }^{17}$ Cronin et al found a correlation between ACA IgM and fetal loss and thrombocytopenia, but no association of ACA IgG with the ACA syndrome. ${ }^{18}$ Also, other investigators 
were unable to find relations between ACAs and thrombosis. ${ }^{15} 16$

Changes in ACA concentrations may be explained by variations in disease activity. In 11/47 patients $(23 \%)$ and the group as a whole ACA IgG was significantly associated with lupus activity. This implies that screening for ACA IgG during lupus flares increases the chance for finding positive concentrations. Our results, however, indicate that during active disease a subset of ACA IgG positive patients with SLE, not characterised by the ACA syndrome, is included. We did not find a relation between ACA IgG and thrombosis using samples obtained during active disease, whereas in the same patients this association was significant during remission. In contrast with ACA-IgG, ACA IgM was found to be significantly associated with lupus activity in only $4 / 47(9 \%)$ patients and not in the group as a whole. In these four patients ACA IgG was also significantly correlated with disease activity. Both during remission and moderate/severe disease activity a significant relation between ACA IgM and thrombosis was found.

We conclude that changes in ACA concentrations are a common phenomenon in patients with SLE, confirming the observations made in two recent reports. ${ }^{8} 19$ Disease activity and prednisone treatment can only partially explain these fluctuations. Associations of ACAs with fetal loss, thrombosis, and thrombocytopenia are mostly established retrospectively and the potential pathogenicity of ACAs for these events has not been proved. Prospective studies are necessary to establish the risks of ACA positive patients with SLE developing the ACA syndrome. As positive ACA titres found in patients with SLE may have consequences on treatment-for example, during pregnancy-we suggest that screening for ACAs should take place in at least two samples taken during low dose prednisone treatment or in its absence. This will increase the chance of finding ACA positive patients possibly at risk. When these samples are obtained during active disease positive ACA IgG concentrations are of limited value in identifying a subset of patients with SLE with clinical events associated with the ACA syndrome. It seems that the IgM isotype is more a marker of this syndrome during lupus flares and we suggest that in active disease ACA IgM should be considered first in evaluating possible future risks for the development of the ACA syndrome.

This work was supported by grants from the Praeventiefonds (28-1511) and the Nederlandse Vereniging voor Reumabestrijding (87 CR 19). The authors would like to thank Drs E J ter Borg and
C G M Kallenberg, University Hospital Groningen, The Netherlands, for their collaboration in this study.

\section{References}

1 Harris E N, Gharavi A E, Boey M L, et al. Anticardiolipin antibodies: detection by radioimmunoassay and association with thrombosis in systemic lupus erythematosus. Lancet 1983; ii: $1211-4$

2 Derksen R H W M, Kater L. Lupus anticoagulant: revival of an old phenomenon. Clin Exp Rheumatol 1985; 3: 349-57.

3 Feinstein D I. Lupus anticoagulant, thrombosis and fetal loss. $N$ Engl J Med 1985; 313: 1348-50.

4 Derue G J, Englert H J, Harris E N, et al. Fetal loss in systemic lupus: association with anticardiolipin antibodies. $J$ Obstet Gynecol Neonatal Nurs 1985; 5: $207-9$.

5 Harris E N, Chan J K H, Asherson R A, Aber V R, Gharavi A E, Hughes G R. Thrombosis, recurrent fetal loss and throm- $\infty$ bocytopenia: predictive value of the anticardiolipin antibody $\vec{N}$ test. Arch Intern Med 1986; 146: 2153-6.

6 Hughes G R V, Harris E N, Gharavi A E. The anticardiolipin $\vec{\circ}$ syndrome. $J$ Rheumatol 1986; 13: 486-9.

7 Isenberg D A, Colaco C B, Dudeney C, Todd-Pokropek A, 0 Snaith M L. The relationship of anti-DNA antibody idiotypes and anticardiolipin antibodies to disease activity in systemic lupus erythematosus. Medicine (Baltimore) 1986; 65: 46-55.

8 Shergy W J, Kredich D W, Pisetsky D S. The relationship of

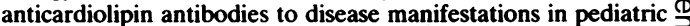
systemic lupus erythematosus. J Rheumatol 1988; 15: 1389-94.

9 Hedfors E, Lindahl G, Lindbald S. Anticardiolipin antibodies during pregnancy. J Rheumatol 1987; 14: 160-1.

10 Derksen R H W M, Biesma D, Bouma B N, Gmelig Meyling F H J, Kater L. Discordant effects of prednisone on antcardiolipin antibodies and the lupus anticoagulant. Arthrifs Rheum 1986; 29: 1295-6.

11 Tan E M, Cohen A S, Fries J F, et al. The 1982 revised criteria for the classification of SLE. Arthritis Rheum 1982; 25: 1271-7.

12 Loizou S, McCrea J D, Rudge A C, Reynolds R, Boyle C C, ㄱ Harris E N. Measurement of anticardiolipin antibodies by an $\frac{\text { O }}{8}$ enzyme-linked immunosorbent assay (ELISA): standardization $\varrho$ and quantitation of results. Clin Exp Immunol 1985; 60: 738-45. $\overrightarrow{\vec{F}}$

13 Derksen R H W M, Hasselaar P, Blokzijl L, Gmelig Meyling F H J, de Groot P G. Coagulation screen is more specific than the anticardiolipin antibody ELISA in defining a thrombotic subset of lupus patients. Ann Rheum Dis 1988; 47: 364-71.?

14 Hazeltine M, Rauch J, Danoff D, et al. Antiphospholipid antibodies in systemic lupus erythematosus. Evidence of an $\bar{\varrho}$ association with positive Coombs' and hypocomplementemia. J Rheumatol 1988; 15: 80-6.

15 Fort J G, Cowchock F S, Abruzzo J L, Bruce Smith J. Anticardiolipin antibodies in patients with rheumatic diseases. Arthritis Rheum 1987; 30: 752-60.

16 Sturfelt G, Nived O, Norberg R, Thorstensson R, Krook K. Anticardiolipin antibodies in patients with systemic lupus $\triangle$ erythematosus. Arthritis Rheum 1987; 30: 382-8.

17 Weidmann C E, Wallace D J, Peter J B, Knight P J, Bear M B. Klinenberg J R. Studies of IgG, IgM and IgA antiphospholipid antibody isotypes in systemic lupus erythematosus. $J$ Rheumatolos 1988; 15: 74-9.

18 Cronin M E, Biswas R B, Van der Straeton C, et al. IgG and IgM anticardiolipin antibodies in patients with lupus with anticardiolipin antibody associated clinical syndromes. $\sigma$ J Rheumatol 1988; 15: 795-8.

19 Kalunian K C, Peter J B, Middlekauff H R, et al. Clinicako significance of a single test for anti-cardiolipin antibodies in $\overline{\mathbb{D}}$ patients with systemic lupus erythematosus. Am J Med 1988; 85: 602-8. 